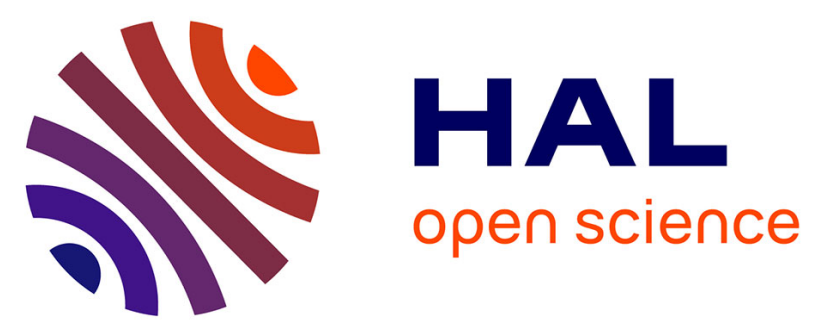

\title{
Analysis and artifact correction for volume correlation measurements using tomographic images from a laboratory X-ray source
}

Nathalie Limodin, Julien Réthoré, Jérôme Adrien, Jean-Yves Buffiere, François Hild, Stéphane Roux

\section{To cite this version:}

Nathalie Limodin, Julien Réthoré, Jérôme Adrien, Jean-Yves Buffiere, François Hild, et al.. Analysis and artifact correction for volume correlation measurements using tomographic images from a laboratory X-ray source. Experimental Mechanics, 2011, 51 (6), pp.959-970. 10.1007/s11340-010-9397-4 . hal-00521190

\section{HAL Id: hal-00521190 \\ https://hal.science/hal-00521190}

Submitted on 26 Sep 2010

HAL is a multi-disciplinary open access archive for the deposit and dissemination of scientific research documents, whether they are published or not. The documents may come from teaching and research institutions in France or abroad, or from public or private research centers.
L'archive ouverte pluridisciplinaire HAL, est destinée au dépôt et à la diffusion de documents scientifiques de niveau recherche, publiés ou non, émanant des établissements d'enseignement et de recherche français ou étrangers, des laboratoires publics ou privés. 
Experimental Mechanics manuscript No.

(will be inserted by the editor)

\title{
Analysis and artifact correction for volume correlation
}

measurements using tomographic images from a laboratory

X-ray source

\author{
Nathalie Limodin Julien Réthoré Jérôme \\ Adrien Jean-Yves Buffière François Hild \\ S. Roux \\ Received: date / Accepted: date
}

\begin{abstract}
N. Limodin, J. Adrien, J.-Y. Buffière
Laboratoire Matériaux, Ingénierie et Sciences (MATEIS)

INSA-Lyon / UMR CNRS 5510

7, avenue Jean Capelle, F-69621 Villeurbanne, France

E-mail: \{nathalie.limodin,jerome.adrien,jen-yves.buffiere\}@insa-lyon.fr

J. Réthoré

Laboratoire de Mécanique des Contacts et des Structures (LaMCoS)

INSA-Lyon / UMR CNRS 5259

20 avenue Albert Einstein, F-69621 Villeurbanne, France

E-mail: julien.rethore@insa-lyon.fr

F. Hild, S. Roux

Laboratoire de Mécanique et Technologie (LMT-Cachan)

ENS Cachan / CNRS / UPMC / PRES UniverSud Paris

61 Avenue du Président Wilson, F-94235 Cachan Cedex, France

${ }^{\star}$ corresponding author

E-mail: \{francois.hild,stephane.roux\}@lmt.ens-cachan.fr
\end{abstract}

Abstract The effects of three artifacts (reconstruction, beam hardening and temperature of the X-ray tube) associated with the use of a lab tomograph are analyzed in terms of their induced biases for Digital Volume Correlation (DVC) from a series of reconstructed volumes acquired successively. The most detrimental effect is due to spurious dilatational strains induced by temperature variations in the tomograph. If they 
are not accounted for, any quantitative kinematic measurement is impossible for strain levels below $0.5 \%$.

Keywords Quantitative kinematic measurements - Reconstruction artifacts . Thermal expansion · X-ray computed microtomography

\section{Introduction}

To visualize inside opaque materials, X-ray computed microtomography (XCMT) has become more and more popular since its invention more than thirty years ago [1,2]. 3D views of various materials [3,4] are obtained in a non-destructive way. Reconstructed volumes give invaluable indications on the structure of metallic [5], ceramic [6,7], cementitious [8], cellular [9] materials. Damage mechanisms [10,11] and cracks [12,13] are also analyzed through direct visualization.

It is also possible to load samples in situ [14]. An additional analysis consists in using the reconstructed volumes themselves to evaluate displacements. The latter are for instance measured by resorting to marker tracking [15]. Another technique consists in local correlations $[16,17]$, in which small interrogation volumes in two scans are registered [18]. An alternative route to local approaches is provided by global correlation techniques $[19,20,21,22]$. The latter will be used herein. In all these cases, the kinematic analysis relies on scans taken at different times. Consequently, they may be corrupted by various phenomena such as reconstruction artifacts, beam hardening, and (for a cone-beam geometry) uncontrolled motion of the source with respect to the sample due to thermal expansion of the set-up during operation. This is the case of lab tomographs.

In Section 2, the main causes of artifacts related to tomography are discussed. Section 3 introduces the basic principles of volume correlation. The way the measured displacements are post-processed to evaluate the effect of different artifacts is discussed. Section 4 is devoted to the effect of temperature variations of the X-ray tube on measured displacements. In Section 5, the effect of reconstruction (so-called ring) artifacts on the performance in terms of standard displacement uncertainty is assessed. 
The influence of beam hardening corrections are studied in Section 6. Last, all these effects are finally considered in Section 7 when a tensile test on nodular graphite cast iron is performed. In particular, the extraction of elastic parameters is discussed and correction procedures are validated.

\section{Tomography}

In the present section, the basic principles of X-ray tomographic imaging are briefly summarized (see for example $[23,24]$ for an exhaustive presentation of the technique) and the specificities of a tomography set-up that uses a laboratory micro-focus X-ray source (lab-CT) are analyzed with an emphasis on artifacts arising from this kind of device.

Because tomography is a non destructive technique, the changes in a sample submitted to various experimental environments can be studied in situ with a volume large enough to yield statistically relevant information. Therefore, in the last 15 years, X-ray CT, originally developed for studies in the medical field, has found more and more applications in materials science (see for example [25] for a recent review of various applications). This increasing interest in tomography and the difficulty to have access to synchrotron facilities have prompted for lab-CT development. This tool has now become widespread for materials characterization, and a precise knowledge of its possibilities and limitations is mandatory whenever accurate and quantitative results are sought.

Although various experimental set-ups are used to perform X-ray tomography, the basic principles of the technique remain the same, namely, an X-ray beam is sent onto a sample mounted on a rotator, a series of $N$ radiographs (herein called a scan) corresponding to $N$ angular positions of the sample $\left(360^{\circ}\right.$ rotation in the case of lab$\mathrm{CT}$ ) in the beam is recorded on a detector that is generally a CCD camera in modern tomographs. Those radiographs are used by a reconstruction software to obtain the 3D distribution of the linear X-ray attenuation coefficient $\mu$ within the sample. This distribution forms the $3 \mathrm{D}$ reconstructed volume. The value of $\mu$ depends on the $\mathrm{X}$ - 
ray photon energy as well as on the material density and atomic number so that the contrast in CT images is usually given by differences in absorption between phases or constituents with different compositions or densities.

A lab-scale tomograph is composed of a source, i.e., an X-ray tube (micro- or nanofocus), a sample manipulation system that allows for accurate positioning and motion of the sample ( 3 translations +1 rotation). A $2 \mathrm{D}$ flat panel detector converts X-rays into digital radiographs stored in a computer. The X-ray tube produces a polychromatic and conical beam whose divergence is used to monitor the image magnification by moving the object relative to the source (the magnification equals the source to detector distance divided by the source to sample distance). The spatial resolution is however limited by the size of the focal spot due to a penumbral blurring effect. A smaller spot size is required for high resolution images, at the expense of the delivered flux (inducing longer scan times).

One of the most basic artifact that are found in tomographic images is caused by the reconstruction software using a wrong reconstruction axis. Hence, prior to reconstruction, the position of the projection on the CCD detector of the sample rotation axis has to be determined accurately. The determination of the "actual" position of the rotation axis is usually left to the user who eventually validates visually the software alignment procedure by looking at reconstructed slices.

Another so-called "ring-artifact" is also frequently observed in tomographic images. Among other causes, it may be due to defective elements of the detector (e.g., elements delivering a non-linear signal) or inhomogeneities in the X-ray beam. Such defects appear on each recorded projection of the sample and produce a ring-shaped contrast centered on the rotation axis in the reconstructed image. Because of the higher angular sampling of the sample close to the rotation axis, ring artifacts are more pronounced towards the rotation axis. A flat-field correction routinely applied on the projections using the "offset" and "gain" images recorded at the beginning of the scan is generally not sufficient to erase these artifacts. These rings may be detrimental to subsequent processing, e.g., segmentation, and thus to quantitative image analyses. The techniques 
used to remove or minimize these rings are divided into two categories, namely, i) postprocessing of the reconstructed data, i.e., filtering, but then part of the contrast due to high frequency features can be lost [27], and ii) pre-processing of the data prior to reconstruction, i.e., sinogram processing $[28,29]$.

The polychromatic X-ray beam used by lab-CT equipments is the source of another type of artifact called beam hardening. The polychromatic beam, when traversing a sample, loses the less energetic of its photons that are absorbed, and therefore the mean energy of the transmitted X-ray beam is higher than that of the incident beam. In practice, long ray paths being more attenuating than short ones, an artificial darkening (resp. brightening) at the centre (resp. edges) of the sample is observed on the reconstructed images impeding, for example, a direct conversion of the gray levels in values of the attenuation coefficient $\mu$. Several solutions have been proposed to reduce beam hardening artifacts, the most classical of which consists in using a thin metallic foil (typically $1 \mathrm{~mm}$-thick or less of copper or aluminium sheets) to filter or "preharden" the beam. The price to pay for this filtering is a decrease in X-ray flux that might result in a lower signal to noise ratio.

Imperfect motions of the rotation stage, motions of the specimen (e.g., insufficiently clamped specimens or living specimens) and motions of the X-ray emission spot are also sources of artifacts on reconstructed images. The sample translations that occur during a scan due to mechanical instabilities or inaccuracies of the sample translations are compensated for by processing the projections. For example, in the reconstruction software used in this work, an image compensation routine allows for "manual" corrections to be applied, namely, shifting and scaling of the last projection $\left(360^{\circ}\right)$ is applied by the user until a good match (assessed by eye) is obtained with the first projection $\left(0^{\circ}\right)$. However, no correction is possible for a tilt that would arise from wobble or eccentricity of the rotator or sample mount. Such artifacts are all the more important when the magnification is high. For nanofocus tubes that enable for magnifications up to 1000 , air bearings and piezo elements are preferred for accurate sample rotation and manipulation. 
In lab-CT, only a small fraction of the energy of the electron beam impinging on the $\mathrm{W}$ target is converted into X-rays, the remainder is dissipated into heat. During a scan acquisition, X-rays are continuously emitted for several tens of minutes and the temperature of the X-ray tube may increase by about $10{ }^{\circ} \mathrm{C}$. If several scans are performed in a row, as usually occurring during in situ studies, the X-ray tube has no time to cool down after one scan, and its temperature increases with the number of scans, i.e., with time. The situation is exactly similar in the case of a single long scan, which is often necessary when high resolution (voxel size $<1 \mu \mathrm{m}$ ) is sought. This increase in temperature induces thermal expansion of the X-ray tube assembly. In turn, thermal dilation causes small geometric motions of the X-ray emission point. These motions can occur in the plane perpendicular to the beam or parallel to it. In the latter case, the source to sample distance steadily decreases during the scan and alters the magnification of the recorded radiographs. Although the displacement of the $\mathrm{X}$-ray tube with respect to the sample is very small, its effect on the radiographs is amplified by geometrical magnification.

In practice, therefore, images with resolutions in the micrometer or sub-micrometer range require that the temperature variation and subsequent expansion of the tube are either controlled or corrected prior to reconstruction. Salmon et al. [30] have proposed to correct shifts in the plane perpendicular to the $\mathrm{X}$ ray beam on individual radiographs. The shifts are determined by registering the $n^{t h}$ radiograph of the scan with the corresponding one (same angular position) of a fast scan (a few angular positions) recorded at the end of the experiment. This correction procedure increases the quality of images and has also an effect on the recorded gray level histograms of various samples / materials. The motions of the source along the beam direction are however not considered by the authors. It will be shown in Section 4 that such motions are also to be taken into account when successive reconstructions or scans are to be compared quantitatively.

The experiments reported in this study are performed using a CT system V-Tomex (Phoenix X-ray) fitted with a nanofocus tube (W target) whose acceleration voltage is 
adjusted from 40 to $160 \mathrm{kV}$, and whose focal spot size is tuneable from $6 \mu \mathrm{m}$ to $1 \mu \mathrm{m}$. The material of the present study, i.e., cast iron (see reference [21] for a description of the material), being strongly attenuating, the tomography experiments are carried out with a rather large spot size, i.e., $3.5 \mu \mathrm{m}$, and a $95 \mathrm{kV}$ acceleration voltage to ensure a $10 \%$ transmission of the X-ray beam through the $1.5 \times 1.5 \mathrm{~mm}^{2}$ crosssection of the sample. The specimen is put on the rotating stage (wobble: $\pm 7 \mu \mathrm{rad}$, eccentricity: $\pm 0.4 \mu \mathrm{m}$, uncertainty of angular positioning: $130 \mu \mathrm{rad}$ ) in the tomograph chamber between the X-ray source and an amorphous Si diode array detector of dimensions $1920 \times 1536$ pixels. A set of 900 radiographs is recorded while the sample is rotating, step by step, over $360^{\circ}$ along its vertical axis. When the radiograph is shot, the rotating stage does not move. With an acquisition time per image of $500 \mathrm{~ms}$, one scan lasted about $45 \mathrm{~min}$. A small tensile testing device was used to load the specimen for in situ test [21]. This device uses a glass cylinder to transmit the load between the top and bottom parts of the machine, and therefore imposes a minimum source to specimen distance of $15.9 \mathrm{~mm}$. This condition results in an image voxel size of $3.5 \mu \mathrm{m}$. Reconstruction of the tomographic data is performed with a filtered backprojection algorithm (i.e., an optimized Feldkamp cone-beam algorithm [31]) using datos $\mathrm{x}$-acquisition and reconstruction software developed by Phoenix. It provides a 3D image with a 16-bit deep gray scale (Figure 1).

Although artifacts possibly affecting image reconstructions have been listed above, reconstructed images will be trusted in the sequel (without specific pre-corrections) but compared to each other to evaluate displacements by resorting to volume correlation. Consequences in terms of sinogram pre-corrections, albeit certainly desirable, will not be investigated herein.

\section{Volume Correlation}

A reconstructed volume is represented as a discrete function $f(\mathbf{x})$ giving the gray level at each voxel position $\mathbf{x}$. For the ease of presentation, such functions will be considered as the discrete sampling of a continuous (interpolated) function. Digital volume 
correlation (DVC) consists in registering two volumes with the help of a displacement field to be measured. The conservation of the local texture between the reference $f$ and deformed $g$ volumes is given by

$$
g(\mathbf{x}+\mathbf{u}(\mathbf{x}))=f(\mathbf{x})
$$

where $\mathbf{x}$ is the position vector, and $\mathbf{u}$ the sought displacement vector. The texture conservation hypothesis is not strictly satisfied. In particular, 3D volumes obtained by $\mathrm{XCMT}$ are the results of a numerical reconstruction procedure that induces artifacts listed in Section 2. The difference, $g(\mathbf{x}+\mathbf{u}(\mathbf{x}))-f(\mathbf{x})$, or correlation residuals, quantifies the quality of volume registration.

When two volumes $f$ and $g$ are considered, the measurement problem consists in evaluating $\mathbf{u}$ as accurately as possible. To measure $\mathbf{u}$, the quadratic difference $\varphi^{2}=$ $[g(\mathbf{x}+\mathbf{u}(\mathbf{x}))-f(\mathbf{x})]^{2}$ is integrated over the studied domain $\Omega$

$$
\Phi^{2}=\int_{\Omega} \varphi^{2} \mathrm{~d} \mathbf{x}
$$

and minimized with respect to the unknown degrees of freedom of the measured displacement field. In the following, a $3 \mathrm{D}$ finite element kinematics is selected for the sought displacement field $\mathbf{u}$. The simplest shape functions are used, namely, trilinear polynomials associated with 8-node cube elements (or C8-DVC). More details on the correlation procedure can be found in Ref. [19]. The size of each element is denoted by $\ell$ so that the total number of voxels per element is $\ell^{3}$.

DVC is used in order to extract significant mechanical quantities from experiments $[32,21,22]$. In the present study this tool is utilized to analyze and possibly correct for artifacts due to reconstruction and/or temperature variations of the X-ray tube. In the following sections two types of analyses are used:

- when a rigid body motion is applied to the sample, the mean and root mean square (RMS) of the displacement field are assessed. The standard displacement uncertainty is then interpreted as a global evaluation of the measurement uncertainty and some additional effects that call for corrections, whenever possible, 
- once the effect of an artifact has been included, or when the actual displacement is not simply a rigid body translation, corrections are proposed to reduce measurement "errors." The measured displacement is then projected onto a basis that contains the rigid body motion and the suspected field associated with an artifact

$$
\mathbf{u}_{i}(\mathbf{x})=\mathbf{u}_{0}+\mathbf{x} \times \boldsymbol{\omega}+\epsilon \mathbf{x}
$$

where $\mathbf{u}_{i}$ is the interpolating displacement field, $\mathbf{u}_{0}$ the mean translation, $\boldsymbol{\omega}$ the corresponding rotation vector, and $\epsilon$ the dilatational strain component. After the contribution of each of these elementary displacements has been calculated by means of a least squares minimization, the RMS of the difference between measured and interpolating displacement fields quantifies the effect of the proposed correction.

This strategy is successively applied to quantify and further correct the effect of temperature variations of the X-ray tube, ring artifacts, and beam hardening.

\section{Temperature variations of the X-ray tube}

In the present operating conditions, acquiring a complete scan of a sample lasts typically 45 minutes. If several scans are acquired successively, X-rays are emitted for about an entire day so that the temperature of the tube may vary by several tens of Celsius degrees from the first to the last scan. Variations of magnification between each radiograph, and more importantly between two scans are therefore expected. This may impact quantitatively the procedures of tomographic image acquisition with a lab tomograph that is not running $24 \mathrm{~h} /$ day.

The following analysis is proposed to quantify this effect. A series of scans of the same unloaded sample are acquired successively. The sample was not moved. The displacement fields with respect to the very first scan are measured by using DVC and further processed. Due to the variation of the magnification induced by the temperature variation of the X-ray tube, an homothetic transformation is looked for. The measured displacement field is thus projected in a least-squares sense onto a basis that contains the rigid body motions (i.e., three translations, and three rotations) and a 
(spurious) scalar dilatational strain $\epsilon$ inducing a dilatational displacement field $\mathbf{u}=\epsilon \mathbf{X}$ (see Equation (3)).

As an example, for the last scan of series \#2 (Figure 3a), the following values are obtained

$$
\mathbf{u}_{i}=\underbrace{\left[\begin{array}{c}
-3.8 \\
10.8 \\
-1.8
\end{array}\right]}_{\text {translation }}+\underbrace{\mathbf{x} \times 10^{-5} \cdot\left[\begin{array}{r}
24 \\
4 \\
-2
\end{array}\right]}_{\text {rotation }}+\underbrace{2.8 \cdot 10^{-3} \mathbf{x}}_{\text {dilation }}
$$

with $\mathbf{x}=\mathbf{O M}, O$ being the center of the region of interest, and $M$ a current point (the positions and displacements are expressed in voxels, where 1 voxel $=3.5 \mu \mathrm{m}$, and the rotations in radian). The observed translation may be due to thermal drift of the focal spot [30] or/and mechanical drifts of the sample and the whole mounting stage.

Figure 2 shows the norm of the displacement field when rigid body motions have been removed, the identified spurious dilatational displacement, and the corrected displacement fields. The maximum amplitude of the measured displacement is about 1.8 voxel, which is much higher than the measurement uncertainty (i.e., 0.08 voxel) of DVC that will be assessed in Section 5. Consequently, the dilatational component cannot be considered as a measurement uncertainty but a bias introduced by the temperature variations of the X-ray tube. Further, after projection onto the chosen basis, the relative projection error is equal to $3.5 \%$, this error being defined as the ratio of the $L^{2}$ norm of the difference between the initial displacement and the interpolated displacement over the $L^{2}$ norm of the measured displacement. This low level of the projection error gives additional confidence in the proposed correction.

Figure 3a shows the change of the estimated dilatational strain with time for the considered series. An exponential fit yields consistent results. The dilatational strain depends on the thermal expansion of the X-ray tube, and the temperature of the tube may vary exponentially with time as expected from a first order system described by the heat equation with a loss term [33]

$$
\epsilon(t)=\epsilon_{\infty}[1-\exp (-t / \tau)]
$$


where $\epsilon_{\infty}$ is the steady state correction to be performed when the whole warm-up duration is concerned. Figure 3 a shows that significant levels are reached when kinematic measurements are sought. For instance, for an in situ tensile test the apparent (measured) strains are expressed as

$$
\epsilon_{l}^{a}=\frac{\sigma}{E}+\epsilon \quad, \quad \epsilon_{l}^{t}=-\frac{\nu \sigma}{E}+\epsilon
$$

where $\epsilon_{l}^{a}$ is the apparent longitudinal strain, $\epsilon_{l}^{t}$ the apparent transverse strain, $\sigma$ the tensile stress, $E$ Young's modulus, and $\nu$ Poisson's ratio. If cast iron is concerned as in the present case, the longitudinal elastic strain level of a tensile test under an applied stress of $290 \mathrm{MPa}$ is of the order of $1.8 \cdot 10^{-3}$, and the corresponding transverse strain is equal to $5 \cdot 10^{-4}$. These levels are less than those observed in Figure 3a. If this effect were not corrected for, there would be no way of estimating quantitatively the elastic properties of the considered material.

In some other cases, the reference scan is acquired at time $t_{\Delta}$, later than the beginning of warm-up, so that a strain increment $\Delta \epsilon$ is to be considered

$$
\Delta \epsilon(t)=\epsilon_{\infty}\left[1-\exp \left\{\left(t_{\Delta}-t\right) / \tau\right\}\right]
$$

where $\epsilon_{\infty}$ has to be adjusted depending on the temperature at the time of the reference image. In the present case no air conditioning system was used. It is assumed however that the characteristic time $\tau$ is a constant feature of the system. The parameters $\epsilon_{\infty}$ and $\tau$ are determined by considering the three previous series (Figure 3b). The values of the steady state strain range from $3.7 \cdot 10^{-3}$ to $4.5 \cdot 10^{-3}$. The time $t_{\Delta}$ is equal to 40 min for the second series, and $170 \mathrm{~min}$ for the third series. These values are consistent with the time durations between the beginning of warm-up and when the acquisitions actually started. For series \#3, three scans were performed before the acquisition of the first point of Figure 3a. The time lapse between switching on the X-ray tube and data collection amounts to $173 \mathrm{~min}$. For series \#2, one scan was acquired by another user before the experiment was started (i.e., a time lapse of $40 \mathrm{~min}$ ). Last, the characteristic time is equal to $130 \mathrm{~min}$. This result shows that a warm-up time of about three hours is required to avoid spurious strain levels greater than $10^{-3}$. It is confirmed by analyzing 
the third series (Figure 3a). A warm-up time of about five hours leads to dilatational artifacts less than $4 \cdot 10^{-4}$ in terms of strain levels.

\section{Ring artifacts}

The reconstruction is an anisotropic process that distinguishes axial and radial directions (see Section 2) because of the very principle of tomography. Differences on the uncertainty level along the rotation axis or in an orthogonal plane are expected. To investigate this effect, a sample is translated inside the tomograph by using either the motor-driven rotation stage or manually with the goniometer on which the sample is mounted. Two cases are distinguished, namely, a longitudinal translation (along the rotation axis), and a transverse translation (perpendicular to the rotation axis).

5.1 Translation along the rotation axis

The sample is translated along the rotation axis (using the motor-driven stage) by a value of about 9 voxels. When the measured displacement field is interpolated, two or three contributions given in Equation (3) are considered. Figure 4 shows the change of standard displacement uncertainties prior to and after corrections as a function of the size $\ell$ of the elements chosen for C8-DVC. For each case, the standard displacement uncertainty is a decreasing function of the element size. When compared with the a priori analysis for which translations are applied artificially on a ROI of $384 \times 384 \times 384$ voxels (using a cubic spline interpolation of gray levels [19,22]), the uncertainty level is significantly higher (Figure 4). Further, even though a decreasing power law is obtained in ideal cases, a saturation of the uncertainty is observed in the experimental case.

From the raw uncertainty curve (i.e., without any rigid body and dilation corrections), the correlation error is predominant for small element sizes (less than 12 voxels). The latter arises from the fact that image correlation is an ill-posed problem, meaning that smaller elements give less regularization and thus higher uncertainty levels are observed [19]. For element sizes greater than 12 voxels, the influence of tomography 
artifacts is dominant. Consequently, the standard displacement uncertainty becomes independent of the element size.

After correcting for the dilatational strain, the saturation level of the uncertainty is lower but still present. If the rotations are also accounted for, a further decrease of the saturation is obtained. The rotation correction does not affect the $z$-component $(z$ being the rotation axis) but only $x$ - and $y$-components. It therefore seems that ring artifacts make the correlation algorithm detect a rotation about the rotation axis. It is confirmed by the values of the identified rotation components when 16-voxel elements are chosen

$$
\boldsymbol{\omega}=10^{-5} \cdot\left[\begin{array}{c}
-4 \\
-2 \\
40
\end{array}\right] \mathrm{rad}
$$

This value is significantly greater than the repositioning error (of the order 13 . $\left.10^{-5} \mathrm{rad}\right)$.

To validate this whole analysis, in particular the resolution in terms of the degrees of freedom associated with the interpolation given in Equation (3), the displacement uncertainty $\sigma_{u}$ associated with each node of 16 -voxel elements (i.e., $\ell=16$ voxels) is of the order of 0.08 voxel (Figure 4). By interpolating the measured displacement field, it is possible to evaluate an order of magnitude of the uncertainties associated with a rotation component, say $\omega_{z}$, and that of $\epsilon$. In the present case, the ROI is a square containing $2 n+1$ nodes (with $n=12$ ). By neglecting the correlations between measured degrees of freedom [34], the rotation uncertainty $\sigma_{\omega}$ becomes

$$
\sigma_{\omega}=\frac{\sigma_{u}}{\sqrt{\sum_{i, j, k}\left(x_{i}^{2}+y_{j}^{2}\right)}}
$$

and that associated with $\epsilon$

$$
\sigma_{\epsilon}=\frac{\sigma_{u}}{\sqrt{\sum_{i, j, k}\left(x_{i}^{2}+y_{j}^{2}+z_{k}^{2}\right)}}
$$

When $-n \leq i, j, k \leq n, \sum_{i, j, k} x_{i}^{2}=\sum_{i, j, k} y_{j}^{2}=\sum_{i, j, k} z_{k}^{2}=n(n+1)(2 n+1)^{3} \ell^{2} / 3$. Consequently, when $\sigma_{u}=0.08$ voxel and $n=12, \sigma_{\omega} \approx 4 \cdot 10^{-6} \mathrm{rad}$, and $\sigma_{\epsilon} \approx$ 
$3.2 \cdot 10^{-6}$. These levels show that the values found above are significantly higher than the resolution of the measurement technique. They are therefore deemed trustworthy.

5.2 Translation perpendicular to the rotation axis

In the same spirit as above, a translation orthogonal to the rotation axis is applied by the operator using the manual goniometer. Figure 5 shows that without correction or by correcting for the dilation only, the uncertainty level is higher than in the previous case. By looking at the correlation residuals (Figure 6), two sets of rings are observed, which means that the correlation fails at achieving a perfect match between the two images in the regions where ring artifacts appear. This is caused by the fact that the position of the rotation axis is different in the sample before and after its motion. It is worth mentioning that ring artifacts are observed in the original image (Figure 1), but they appear more clearly on the correlation residuals. After rotation correction, the uncertainty level is similar to that observed in the previous case. This means that the spurious rotation is even more pronounced in the case of a translation orthogonal to the rotation axis. The rotation correction has now an influence on three components, which is understandable from the identified rotation for 16 -voxel elements

$$
\boldsymbol{\omega}=10^{-5} \cdot\left[\begin{array}{c}
-28 \\
+68 \\
17
\end{array}\right] \mathrm{rad}
$$

Note that even with artifact corrections, the uncertainty evaluation that could have been performed using an artificial translation of the image still underestimates the true uncertainty of the measurement (about three times, see Figures 4 and 5). Yet, without corrections, this ratio increases to more than one decade. A significant part of that offset can be attributed to the very nature of the (reconstructed) volumes and their associated artifacts when compared to the performances observed with $2 \mathrm{D}$ pictures $[35$, $36,37]$.

From the analyses of the present section, it is concluded that in the experimental conditions of this work, the displacement uncertainty for 16-voxel elements is less than 
0.08 voxel. The standard uncertainty of the mean normal strain per element is less than $3.5 \cdot 10^{-3}$, that of an average rotation per element is less than $2.5 \cdot 10^{-3} \mathrm{rad}$. Last, for the considered ROI containing $24^{3}$ elements, $\sigma_{\omega}<4 \cdot 10^{-6} \mathrm{rad}$, and $\sigma_{\epsilon}<3.2 \cdot 10^{-6}$.

\section{Beam hardening}

As illustrated by Figure 7, beam hardening affects the image reconstruction on the boundary of the sample. This can be corrected during the reconstruction process. Its influence can also be seen on the gray level histogram. In the present paper, the software that is provided with the tomograph is used as a "black box." In Figure 7, the effect of the beam hardening correction (BHC) is clearly observed. The image is sharper and some perturbations on the histogram have been erased.

To quantify the effect of the beam hardening correction, the uncertainty study is performed only for the longitudinal translation. The results are shown in Figure 8. The standard displacement uncertainty are compared before dilatational strain correction, rotation correction when the analysis is performed with reconstructed volumes with or without the beam hardening correction. It is concluded that the beam hardening correction has a negligible effect on the displacement uncertainty.

\section{Strain measurement}

Last, an in situ tensile test is performed on a smooth sample. The strains that are applied to the specimen are sought. The previous procedure is applied again. If an infinitesimal strain tensor is directly searched for (in addition to translations and rotations) by considering the raw displacements, the following results are obtained

$$
\boldsymbol{\varepsilon}=10^{-3} \cdot\left[\begin{array}{lll}
3.4 & 0.0 & 0.2 \\
0.0 & 3.3 & 0.4 \\
0.2 & 0.4 & 4.8
\end{array}\right]_{\mathbf{i}, \mathbf{j}, \mathbf{k}}
$$

while the relative projection error is 1 percent. The loading axis being aligned with $\mathbf{k}$, this result is completely erroneous. Due to the linear dependence between the mechani- 
cal basis and the dilation basis, the correction and fit are performed in two steps. First, a projection is computed onto a basis that includes three translations, three rotations, the artificial dilatational strain and a displacement field describing a uniaxial tensile test

$$
\boldsymbol{\varepsilon}=\left[\begin{array}{ccc}
-\nu & 0 & 0 \\
0 & -\nu & 0 \\
0 & 0 & 1
\end{array}\right]_{\mathbf{i}, \mathbf{j}, \mathbf{k}}
$$

where $\nu$ is Poisson's ratio of the material. For the maximum applied load level, the dilatational strain $\epsilon=3.7 \cdot 10^{-3}$, and the longitudinal strain $\varepsilon_{k k}=1.1 \cdot 10^{-3}$. This first prediction of the longitudinal strain is at least three times less than the dilatational strain, which explains why the first results were wrong.

From this first projection, the dilatational strain is known, and the displacement corrected for this artifact is then projected onto a basis that contains three translations, three rotations and a field corresponding to a uniform strain state. The infinitesimal strain tensor then becomes

$$
\boldsymbol{\varepsilon}=10^{-3} \cdot\left[\begin{array}{ccc}
-0.2 & 0.0 & 0.0 \\
0.0 & -0.4 & 0.2 \\
0.0 & 0.2 & 1.1
\end{array}\right]_{\mathbf{i}, \mathbf{j}, \mathbf{k}}
$$

The identified strain tensor is now consistent with the tensile load that is applied to the sample, the strain derived from the global measurement (i.e., stroke of the crosshead) being $1.0 \cdot 10^{-3}$ for the maximum load level.

Figure 9 shows the change of strains as a function of the applied load. Without correction, the longitudinal strain $\varepsilon_{k k}$ is significantly overestimated due to dilatational effects. Further, the dilatational strain does not depend on the applied load but on time and its effect is thus increasing during the test. This trend is consistent with the observations of Section 4 (Figure 3). After correction of this artifact, there is a very good agreement between the corrected strain and that derived from the global measurements (based upon the crosshead stroke). With the eigen strains of the corrected strain tensor $\varepsilon_{1} \leq \varepsilon_{2}<\varepsilon_{3}$, the identified value of Poisson's ratio is $\nu=-\left(\varepsilon_{1}+\varepsilon_{2}\right) / 2 \varepsilon_{3}=0.27$, which 
is consistent with the known value of 0.28 [38]. Young's modulus is found to be equal to $156 \mathrm{GPa}$ in good agreement with classical values of SG cast iron (160 $\pm 3 \mathrm{GPa}[38])$.

\section{Conclusion}

X-ray computed tomography is a very powerful tool to visualize opaque materials. When the scans are further processed to measure displacement fields using DVC, it has been shown herein that special care should be exercised. This is particularly true for lab equipments in which divergent beams are used.

First, if the temperature of the X-ray tube is not controlled, it may induce distance variations that result in magnification changes and thus spurious dilatational strains whose maximum level (here observed to be of the order of $0.4 \%$ ) impedes any quantitative (and therefore trustful) displacement measurement. In the present case, it was shown that the elastic properties of a cast iron cannot be evaluated without a correction of the displacement field. This correction was also needed when stress intensity factors were sought in similar experiments on cracked samples [21]. In both cases, it allowed for quantitative estimates of mechanical parameters. It should however be emphasized that this artifact also impacts image reconstruction, although this effect has not been considered in the present study.

Second, because of the principle of tomography, reconstruction (ring) artifacts are observed. It was shown that they degrade the overall performance in terms of measurement uncertainties whose level is close to the decivoxel range, rather than the centipixel range when optical images are used [35,36,37]. Consequently, the identification of elastic properties becomes more delicate, even though possible as shown herein when analyzing a tensile test on cast iron.

Third, in lab tomographs polychromatic beams are usually used. They lead to socalled beam hardening effects. Commercial tomographs are generally provided with correction procedures whose details are not given to the users. In the present case, it was shown that in terms of displacement uncertainty, there is no need to correct the reconstructed volumes. 
Last, to quantify all the previous effects, the procedure followed herein is generic and can be considered as a baseline analysis to better characterize a new equipment, or an existing one when quantitative analyses such as displacement measurements are performed. Among the three effects studied herein, the spurious dilatational strains are definitely the most important to correct.

\section{Acknowledgments}

This work was funded by Agence Nationale de la Recherche under the grant ANR-09BLAN-0009-01 (RUPXCUBE Project). 


\section{References}

1. J. Ambrose and G. N. Hounsfield, Computerized transverse axial tomography, Br. J. Radiol. 46 [542] (1973) 148-149.

2. G. N. Hounsfield, Computerized transverse axial scanning (tomography). 1. Description of system, Br. J. Radiol. 46 [552] (1973) 1016-22.

3. J. Baruchel, J.-Y. Buffière, E. Maire, P. Merle and G. Peix, X-Ray Tomography in Material Sciences, (Hermes Science, Paris (France), 2000).

4. D. Bernard, edt., 1st Conference on 3D-Imaging of Materials and Systems 2008, (ICMCB, Bordeaux (France), 2008.

5. O. Ludwig, M. Dimichiel, L. Salvo, M. Suéry and P. Falus, In-situ Three-Dimensional Microstructural Investigation of Solidification of an Al-Cu Alloy by Ultrafast X-ray Microtomography, Metall. Mat. Trans. A 36 [6] (2005) 1515-1523.

6. E. Maire, P. Colombo, J. Adrien, L. Babout and L. Biasetto, Characterization of the morphology of cellular ceramics by 3D image processing of X-ray tomography, J. Eur. Ceram. Soc. 27 (2007) 1973-1981.

7. T. Juettner, H. Moertel, V. Svinka and R. Svinka, Structure of kaoline-alumina based foam ceramics for high temperature applications, J. Eur. Ceram. Soc. 27 (2007) 1435-1441.

8. T. J. Chotarda, A. Smith, M. P. Boncoeur, D. Fargeot and C. Gault, Characterisation of early stage calcium aluminate cement hydration by combination of non-destructive techniques: acoustic emission and X-ray tomography, J. Eur. Ceram. Soc. 23 (2003) 22112223.

9. E. Maire, A. Fazekas, L. Salvo, R. Dendievel, S. Youssef, P. Cloetens and J. M. Letang, Xray tomography applied to the characterization of cellular materials. Related finite element modeling problems, Comp. Sci. Tech. 63 [16] (2003) 2431-2443.

10. L. Babout, E. Maire, J.-Y. Buffière and R. Fougères, Characterisation by X-Ray computed tomography of decohesion, porosity growth and coalescence in model metal matrix composites, Acta Mater. 49 [11] (2001) 2055-2063.

11. J. Bontaz-Carion and Y.-P. Pellegrini, X-Ray Microtomography Analysis of Dynamic Damage in Tantalum, Adv. Eng. Mat. 8 [6] (2006) 480-486.

12. R. Sinclair, M. Preuss, E. Maire, J.-Y. Buffière, P. Bowen and P. J. Withers, The effect of fibre fractures in the bridging zone of fatigue cracked Ti6Al4V/SiC fibre composites, Acta Mater. 52 [6] (2004) 1423-1438.

13. E. Ferrié, J.-Y. Buffière, W. Ludwig, A. Gravouil and L. Edwards, Fatigue crack propagation: In situ visualization using X-ray microtomography and $3 \mathrm{D}$ simulation using the extended finite element method, Acta Mat. 54 [4] (2006) 1111-1122. 
14. J.-Y. Buffière, E. Ferrié, H. Proudhon and W. Ludwig, Three-dimensional visualisation of fatigue cracks in metals using high resolution synchrotron X-ray micro-tomography, Mat. Sci. Tech. 22 [9] (2006) 1019-1024.

15. S. F. Nielsen, H. F. Poulsen, F. Beckmann, C. Thorning and J. A. Wert, Measurements of plastic displacement gradient components in three dimensions using marker particles and synchrotron X-ray absorption microtomography, Acta Mater. 51 [8] (2003) 2407-2415.

16. B. K. Bay, T. S. Smith, D. P. Fyhrie and M. Saad, Digital volume correlation: threedimensional strain mapping using X-ray tomography, Exp. Mech. 39 (1999) 217-226.

17. M. Bornert, J.-M. Chaix, P. Doumalin, J.-C. Dupré, T. Fournel, D. Jeulin, E. Maire, M. Moreaud and H. Moulinec, Mesure tridimensionnelle de champs cinématiques par imagerie volumique pour l'analyse des matériaux et des structures, Inst. Mes. Métrol. 4 (2004) 4388.

18. T. O. McKinley and B. K. Bay, Trabecular bone strain changes associated with subchondral stiffening of the proximal tibia, J. Biomech. 36 [2] (2003) 155163.

19. S. Roux, F. Hild, P. Viot and D. Bernard, Three dimensional image correlation from X-Ray computed tomography of solid foam, Comp. Part A 39 [8] (2008) 1253-1265.

20. J. Réthoré, J.-P. Tinnes, S. Roux, J.-Y. Buffière and F. Hild, Extended three-dimensional digital image correlation (X3D-DIC), C. R. Mécanique 336 (2008) 643-649.

21. N. Limodin, J. Réthoré, J.-Y. Buffière, A. Gravouil, F. Hild and S. Roux, Crack closure and stress intensity factor measurements in nodular graphite cast iron using 3D correlation of laboratory X ray microtomography images, Acta Mat. 57 [14] (2009) 4090-4101.

22. J. Rannou, N. Limodin, J. Réthoré, A. Gravouil, W. Ludwig, M.-C. Baïetto-Dubourg, J.-Y. Buffière, A. Combescure, F. Hild and S. Roux, Three dimensional experimental and numerical multiscale analysis of a fatigue crack, Comp. Meth. Appl. Mech. Eng. 199 (2010) $1307-1325$

23. A. C. Kak and M. Slaney, Principles of computerized tomographic imaging, (IEEE Press, New York, 1988).

24. S. R. Stock, MicroComputed Tomography: Methodology and Applications, (CRC, 2008).

25. S. R. Stock, Recent advances in X-Ray microtomography applied to materials, Int. Mat. Rev. 53 [3] (2008) 129-181.

26. G. R. Davis and J. C. Elliot, Artefacts in X-ray microtomography of materials, Mat. Sci. Eng. 22 [9] (2006) 1011-1018.

27. ESRF, http://www.esrf.eu/UsersAndScience/Experiments/Imaging/ID19/Software/ringcorrection, (accessed on december 15, 2009).

28. M. L. Rivers and Y. Wang, Recent developments in microtomography at GeoSoilEnviroCARS, in: Developments in X-Ray Tomography V, U. Bonse, eds., (SPIE, Bellingham Wa, 2006) 6318 0J-1-15. 
29. R. A. Ketcham, New algorithms for ring artefact removal, in: Developments in X-Ray Tomography V, U. Bonse, eds., (SPIE, Bellingham Wa, 2006), 6318 00-1-15.

30. P. L. Salmon, X. Liu and A. Sasov, A post scan method for correcting artefacts of slow geometry changes during micro-tomographic scans, J. X-Ray Sci. Tech. 17 (2009) 161-174.

31. L. A. Feldkamp, L. C. Davis and J. W. Kress, Practical cone beam algorithm, J. Opt. Soc. Am. A1 (1984) 612-619.

32. F. Hild, E. Maire, S. Roux and J.-F. Witz, Three dimensional analysis of a compression test on stone wool, Acta Mat. 57 (2009) 3310-3320.

33. A. B. De Vriendt, La transmission de la chaleur, (Morin, Québec (Canada), 1987).

34. G. Besnard, F. Hild and S. Roux, "Finite-element" displacement fields analysis from digital images: Application to Portevin-Le Chatelier bands, Exp. Mech. 46 (2006) 789-803.

35. H. W. Schreier, J. R. Braasch and M. A. Sutton, Systematic errors in digital image correlation caused by intensity interpolation, Opt. Eng. 39 [11] (2000) 2915-2921.

36. X. Fayolle, S. Calloch and F. Hild, Controlling testing machines with digital image correlation, Exp. Tech. 31 [3] (2007) 57-63.

37. K. Triconnet, K. Derrien, F. Hild and D. Baptiste, Parameter choice for optimized digital image correlation, Opt. Lasers Eng. 47 (2009) 728-737.

38. P. Dierickx, Etude de la microstructure et des mécanismes d'endommagement de fontes G.S. ductiles : influence des traitements thermiques de ferritisation, (PhD dissertation, INSA de Lyon, 1996). 


\section{List of Figures}

1 Schematic view of the process of tomographic acquisition and reconstruction. Ring artifacts are visible on one axial cut of the reconstructed

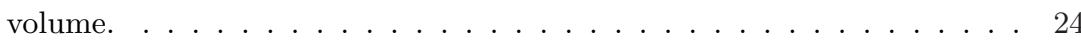

2 Norm in voxels for the measured (a) displacement after removal of rigid body motions, the dilatational displacement (b), and the difference of the two displacement fields (c). The region of interest is cut through its mid-plane for visualization purposes. . . . . . . . . . . . . . 25

3 Change of the dilatational strain with time for three series of experiments (a). Change of the normalized dilatational strain $\epsilon / \epsilon_{\infty}$ with time for the three series (b). The dashed lines correspond to the interpolation by using Equations (5) and (6) . . . . . . . . . . . . . . 26

4 Change of the standard displacement uncertainty when a longitudinal translation is prescribed to the sample. The effect of a correction accounting for dilation, and rotation is compared to the raw data. The result of the a priori analysis is shown for comparison purposes. The curves are linear interpolations (in the log-log plot) of the various results. 27

5 Change of the standard displacement uncertainty when a transverse translation is prescribed to the sample. The effect of a correction accounting for dilation, and rotation is compared to the raw data. The result of the a priori analysis is shown for comparison purposes. The curves are linear interpolations (in the log-log plot) of the various results. 28

6 Correlation residuals for a longitudinal (a) and transverse (b) motion. The ring artifacts are clearly visible, and the locations of the two rotation axes when a transverse motion is applied. . . . . . . . . . . . . 29

7 Illustration of beam hardening affecting image reconstruction. . . . . . . 30 
8 Standard displacement uncertainty versus element size for raw measurements and after the correction with the fields of Equation (4). The same results are obtained with and without beam hardening corrections. The result of the a priori analysis is shown for comparison purposes. The curves are linear interpolations (in the log-log plot) of the various results. 31

9 Strains as functions of the load level for DVC measurements with and without corrections. The dilatational strain is also plotted. Its level is more important than the longitudinal (mechanical) strain. . . . . . . . 32 


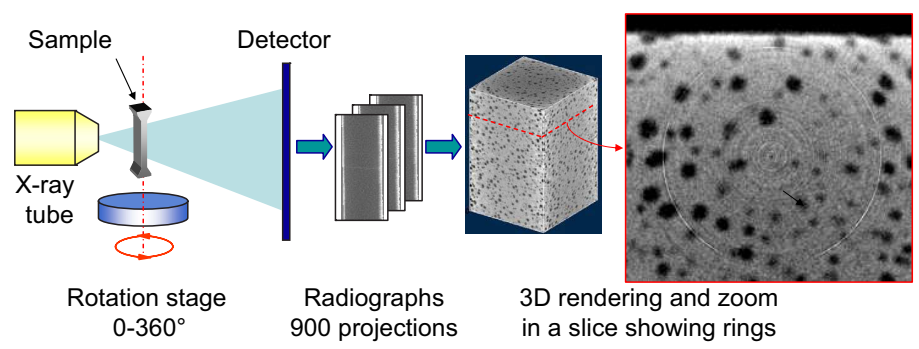

Fig. 1 Schematic view of the process of tomographic acquisition and reconstruction. Ring artifacts are visible on one axial cut of the reconstructed volume. 


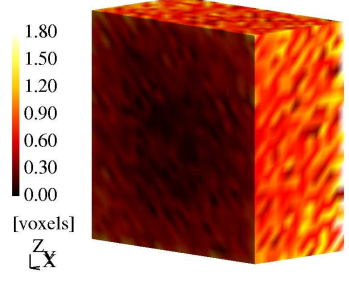

(a)

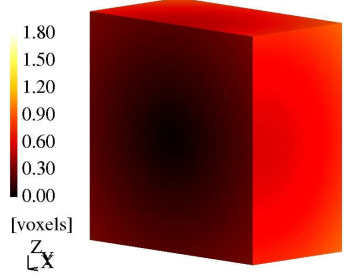

(b)

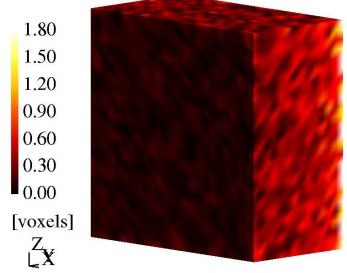

(c)

Fig. 2 Norm in voxels for the measured (a) displacement after removal of rigid body motions, the dilatational displacement (b), and the difference of the two displacement fields (c). The region of interest is cut through its mid-plane for visualization purposes. 


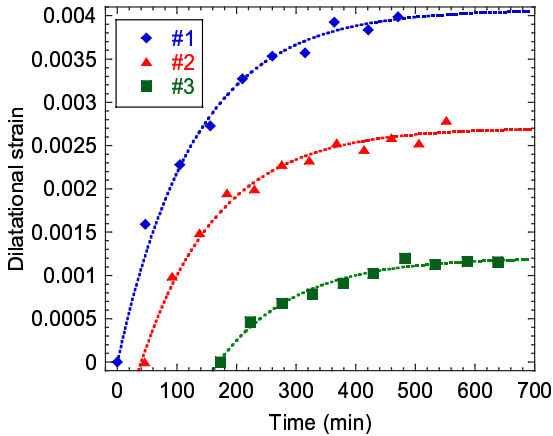

(a)

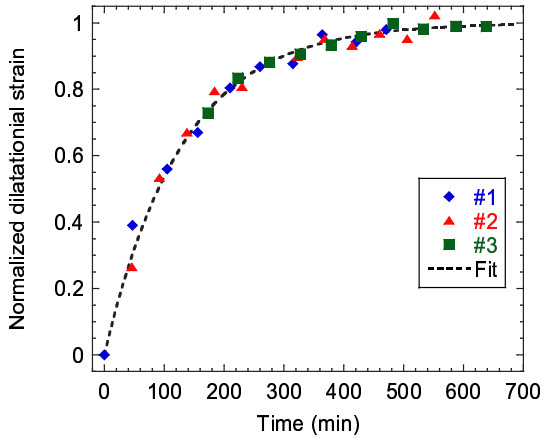

(b)

Fig. 3 Change of the dilatational strain with time for three series of experiments (a). Change of the normalized dilatational strain $\epsilon / \epsilon_{\infty}$ with time for the three series (b). The dashed lines correspond to the interpolation by using Equations (5) and (6). 


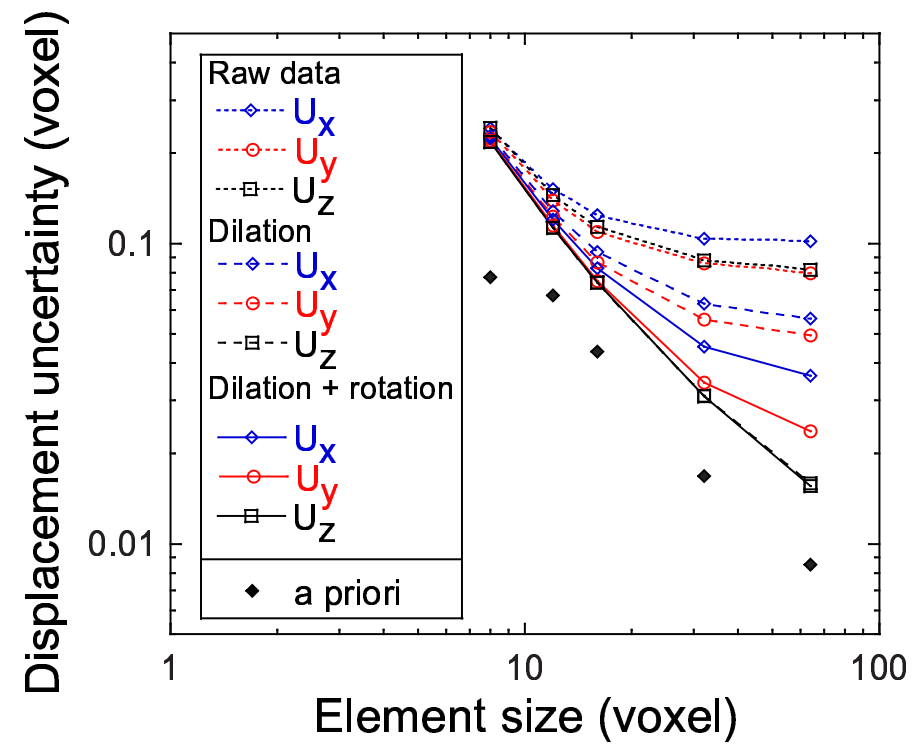

Fig. 4 Change of the standard displacement uncertainty when a longitudinal translation is prescribed to the sample. The effect of a correction accounting for dilation, and rotation is compared to the raw data. The result of the a priori analysis is shown for comparison purposes. The curves are linear interpolations (in the log-log plot) of the various results. 


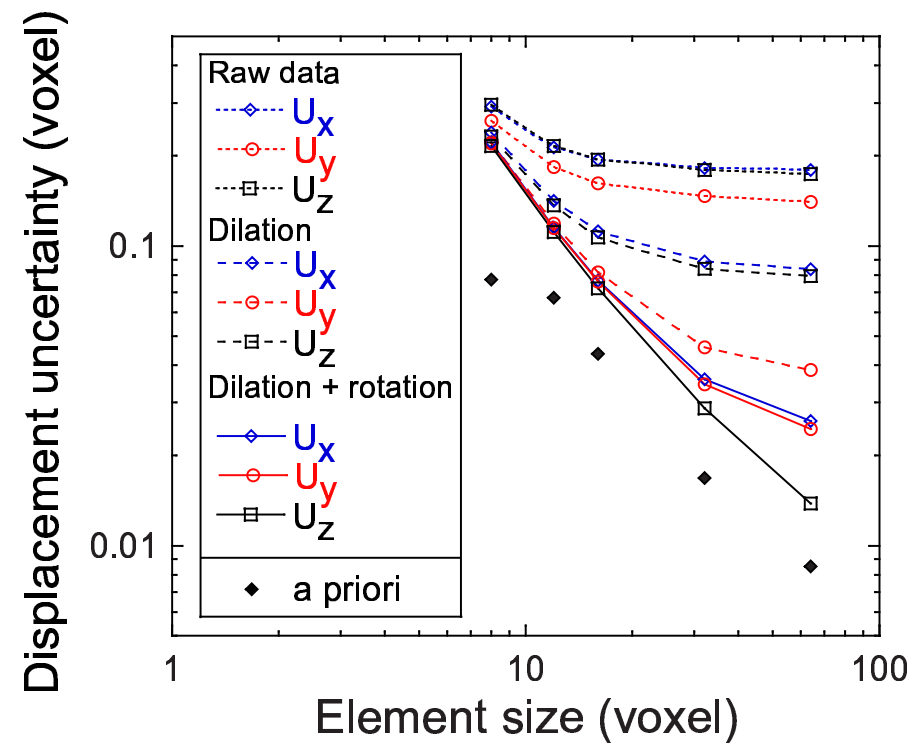

Fig. 5 Change of the standard displacement uncertainty when a transverse translation is prescribed to the sample. The effect of a correction accounting for dilation, and rotation is compared to the raw data. The result of the a priori analysis is shown for comparison purposes. The curves are linear interpolations (in the log-log plot) of the various results. 


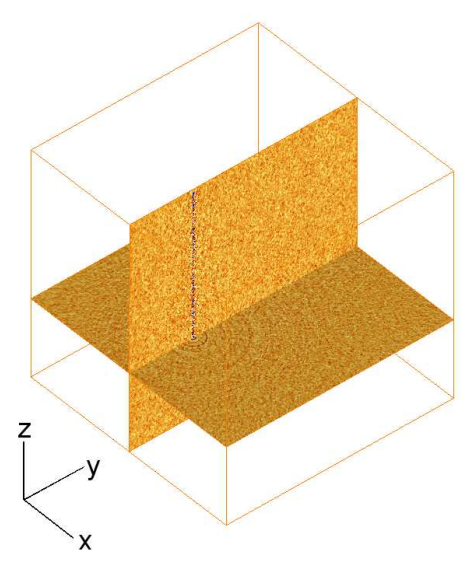

(a)

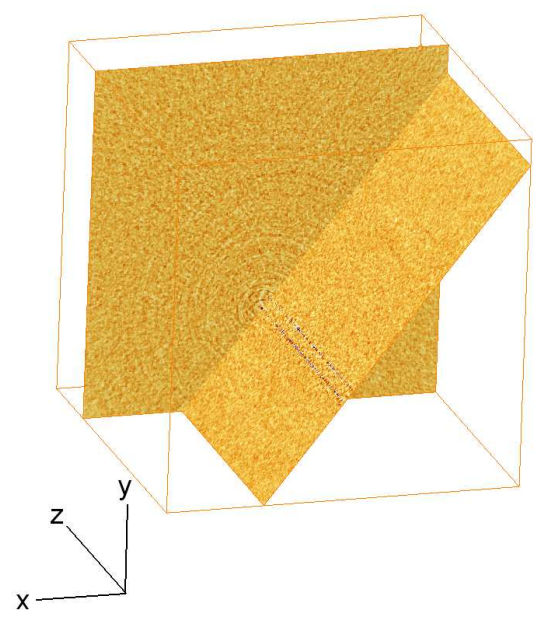

(b)

Fig. 6 Correlation residuals for a longitudinal (a) and transverse (b) motion. The ring artifacts are clearly visible, and the locations of the two rotation axes when a transverse motion is applied. 


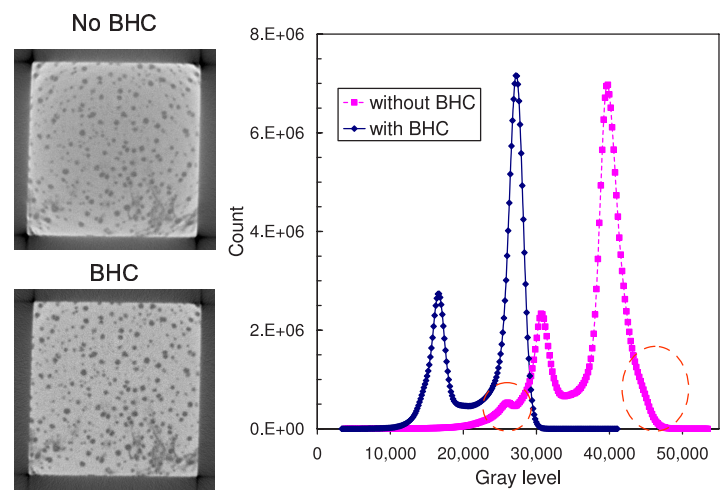

Fig. 7 Illustration of beam hardening affecting image reconstruction. 


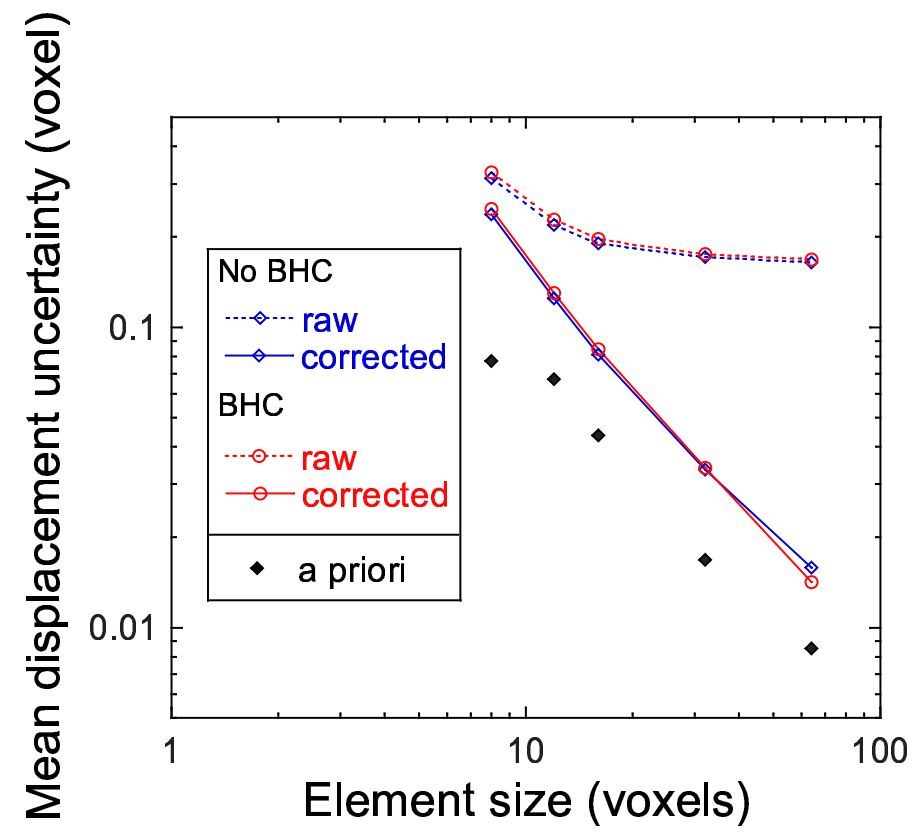

Fig. 8 Standard displacement uncertainty versus element size for raw measurements and after the correction with the fields of Equation (4). The same results are obtained with and without beam hardening corrections. The result of the a priori analysis is shown for comparison purposes. The curves are linear interpolations (in the log-log plot) of the various results. 


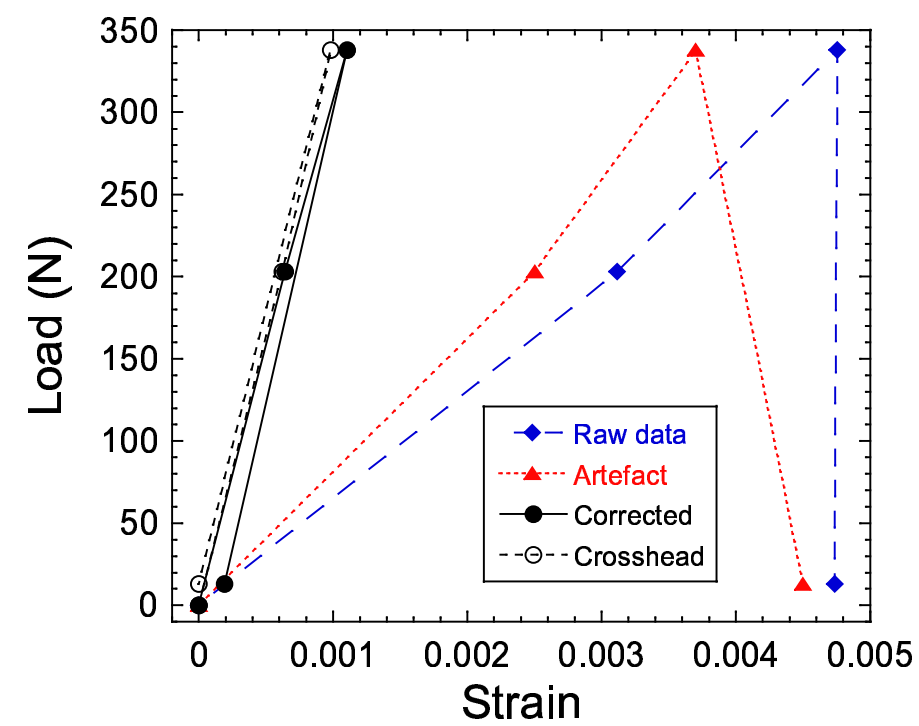

Fig. 9 Strains as functions of the load level for DVC measurements with and without corrections. The dilatational strain is also plotted. Its level is more important than the longitudinal (mechanical) strain. 